Operative Techniken

Oper Orthop Traumatol 2019 $\cdot 31: 293-300$ https://doi.org/10.1007/s00064-019-0611-y Eingegangen: 30. Oktober 2018

Überarbeitet: 20. Januar 2019

Angenommen: 16. April 2019

Online publiziert: 3. Juni 2019

() Der/die Autor(en) 2019

\section{Redaktion}

K. Dresing, Göttingen

Zeichner

R Himmelhan, Mannheim

\section{Vorbemerkungen}

Die Lockerung von Pedikelschrauben ist die häufigste Versagensursache dorsaler Instrumentationen im postoperativen Verlauf [1-3]. Jedoch kann es auch bereits intraoperativ zu einem Versagen der eingebrachten Schrauben kommen. Bei der Reposition von Frakturen oder bei Distraktions-Kompressions-Manövern, wird bereits eine relevante Kraft auf das Schrauben-Knochen-Interface ausgeübt und somit kann es bereits in diesem Stadium zu einer Schraubenlockerung kommen.

Aus biomechanischen Studien ist bekannt, dass Pedikelschrauben bei osteoporotischer/osteopener Knochenstruktur nur noch unzureichend stabil im Wirbelkörper verankert werden können und somit bei einer Knochendichte unter $80 \mathrm{mg} / \mathrm{cm}^{3}$ die Versagenslast im Vergleich zu einem Wirbelkörper mit normaler Knochendichte um 40 \% abfällt [4].

Die Zementaugmentation von Pedikelschrauben wurde bereits vielfach biomechanisch untersucht. Sowohl aus „Pull-out“-Versuchen als auch aus Dauerbelastungstests ist bekannt, dass die Pedikelschraubenstabilität signifikant gesteigert werden kann [5-7]. Auch bei einer Pseudarthrose mit konsekutiv eingetretener Lockerung der einliegenden Instrumentation konnte die Schraubenstabilität mittels nachträglicher Zementaugmentation wiederhergestellt werden [8].

Lukas Weiser - Stephan Sehmisch - Lennart Viezens - Wolfgang Lehmann

Klinik für Unfallchirurgie, Orthopädie und Plastische Chirurgie, Universitätsmedizin Göttingen, Göttingen, Deutschland

\title{
Intraoperative Revision von primär ausgerissenen Pedikelschrauben
}

Bei osteopener/osteoporotischer Knochenqualität kann es, wie oben erwähnt, jedoch bereits intraoperativ $\mathrm{zu}$ einer Pedikelschraubenlockerung kommen. Aus unserer Sicht ist in solchen Fällen eine Zementaugmentation auch noch nachträglich, nach intraoperativ bereits eingetretener Lockerung möglich.

Ein weiteres Verfahren, um eine intraoperativ gelockerte Pedikelschraube erneut stabil im Wirbelkörper zu verankern, ist die Wahl eines größeren Schraubendurchmessers oder die Implantation in einer anderen Schraubentrajektorie [9-12].

\section{Operationsprinzip und -ziel}

Ziel des Eingriffs ist die Revision einer intraoperativ gelockerten Pedikelschraube. Die eingebrachten Pedikelschrauben sollten anschlieBend eine ausreichende Stabilität aufweisen, um eine gegebenenfalls durchgeführte Reposition zu halten, eine Frakturheilung zu gewährleisten oder eine knöcherne Fusion zu ermöglichen.

\section{Vorteile}

- Stabile Verankerung der Pedikelschrauben im Wirbelkörper

- Reduzierung der Komplikationsrate durch Verbesserung der Implantatstabilität

- Einfach zu erlernende und anzuwendende Techniken
- Frühfunktionelle Nachbehandlung des oftmals geriatrischen Patientenkollektivs

\section{Nachteile}

- Allgemeine Operationsrisiken

- Möglichkeit der Implantatfehllage und Verletzung benachbarter Strukturen

- Möglichkeit einer Zementembolie oder einer Zementleckage, anaphylaktischer Reaktionen mit relevanten Kreislaufreaktionen sowie thermischer Schäden [13]

- Symptomatische Komplikationen in 5,5\% der Fälle

- Asymptomatische Zementleckage in $66,7 \%$ der Fälle

- Anaphylaktische Reaktion in 1,2\% der Fälle

- Im Revisionsfall muss von einem höheren Leckagerisiko ausgegangen werden, da der Schraubenkanal erweitert ist und ggf. eine Perforation vorliegt

\section{Indikationen}

- Intraoperativ gelockerte oder ausgerissene Pedikelschrauben

\section{Kontraindikationen}

- Allgemeine Kontraindikationen bezüglich Anästhesie und Operation 


\section{Patientenaufklärung}

- Allgemeine Operationsrisiken

- Verletzungen benachbarter Strukturen z. B. Spinalnerv, Dura, Myelon, Gefäße

- Implantatfehllage, -bruch, -lockerung, -dislokation

- Zementleckage, Zementembolie, allergische Reaktion

\section{Operationsvorbereitungen}

- Ausführliche Anamnese und Untersuchung des Patienten.

- Bestimmung der Knochendichte in den zu instrumentierenden Wirbelkörpern mittels quantitativer Computertomographie (qCT) oder DXA-Messung („dual energy X-ray absorptiometry")

- Planung der Schraubenlänge und -dicke anhand der vorhandenen Bildgebung

\section{Instrumentarium}

- Pedikelschraubensystem

- Bildwandler

- Bei Zementaugmentation kanülierte, fenestrierte Schrauben und Augmentationskit mit Knochenzement

\section{Anästhesie und Lagerung}

- Vollnarkose

- Bauchlage auf einem röntgendurchlässigen Tisch

- Während der Zementaugmentation Erhöhung des PEEP („positive endexpiratory pressure") zur Prävention einer Zementleckage

Oper Orthop Traumatol 2019 · 31:293-300 https://doi.org/10.1007/s00064-019-0611-y

(c) Der/die Autor(en) 2019

L. Weiser · S. Sehmisch · L. Viezens · W. Lehmann

Intraoperative Revision von primär ausgerissenen Pedikelschrauben

Zusammenfassung

Operationsziel. Revision von intraoperativ ausgerissenen Pedikelschrauben.

Indikationen. Intraoperativ gelockerte/ausgerissene Pedikelschrauben. Kontraindikationen. Keine.

Operationstechnik. Entfernung der gelockerten/ausgerissenen Pedikelschraube. Austasten des Schraubenkanals und Reimplantation unter Verwendung einer anderen Trajektorie, eines größeren Schraubendurchmessers oder einer zementaugmentierten Pedikelschraube.

Weiterbehandlung. Frühfunktionelle Mobilisation und ggf. Einleitung einer Osteoporosetherapie.

Ergebnisse. In einer biomechanischen Studie an humanen Kadaverwirbelkörpern erfolgte ein Dauerbelastungstest mit augmentierten sowie nichtaugmentierten Pedikelschrauben. Nach eingetretener Lockerung wurden eine nachträgliche Augmentation der gelockerten, nichtaugmentierten Schrauben und ein erneuter Dauerbelastungstest durchgeführt. Sowohl die initiale $(p=0,009)$ als auch die Augmentation nach Lockerung $(p=0,001)$ zeigten einen signifikanten Anstieg der Versagenslast verglichen mit den nichtaugmentierten Pedikelschrauben. In unserem eigenen Patientenkollektiv aus dem Zeitraum April 2016 bis August 2018 zeigte sich bei 11 von 542 mit Pedikelschrauben versorgten Patienten eine intraoperative Schraubenlockerung. Diese wurde in 6 Fällen mit einer nachträglichen Augmentation und in 5 Fällen mit einem dickeren Schraubendurchmesser revidiert. In der postoperativen Kontrolle nach 6 Wochen zeigte sich keine dieser Schrauben erneut gelockert.

Schlüsselwörter

Knochenschraube $\cdot$ Knochendichte .

Knochenzement · Osteoporose · Postoperative Komplikationen

\section{Intraoperative revision of initially loosened pedicle screws}

\section{Abstract}

Objective. Intraoperative revision of initially loosened pedicle screws.

Indications. Intraoperatively loosened/stripped pedicle screws.

Contraindications. None.

Surgical technique. Removal of the loosened/stripped pedicle screw. Checking the screw channel and re-implantation using a different trajectory, a larger screw diameter or a cement-augmented pedicle screw. Postoperative management. Early functional mobilization and initiation of osteoporosis therapy if indicated.

Results. A biomechanical study with human vertebral bodies was performed. Augmented and not augmented pedicle screws were tested until loosening using a fatigue testing setup. After loosening occurred a subsequent augmentation of the loosened, not augmented screw was performed, and it was tested using a fatigue test again. Both the initial $(p=0.009)$ and the augmentation after loosening $(p=0.001)$ showed a significant increase in failure load compared to the nonaugmented pedicle screws. In our own patient collective from April 2016 to August 2018, 11 of 524 patients treated with pedicle screws showed intraoperative screw loosening. This was revised in 6 cases with a subsequent augmentation and in 5 cases with a larger screw diameter. In the postoperative control at 6 weeks, none of these screws showed loosening again.

\section{Keywords}

Bone screws · Bone mineral density · Bone cement - Osteoporosis · Postoperative complications 


\section{Operationstechnik}

(-Abb. 1, 2, 3, 4, 5, 6).

Diagnose der Schraubenlockerung
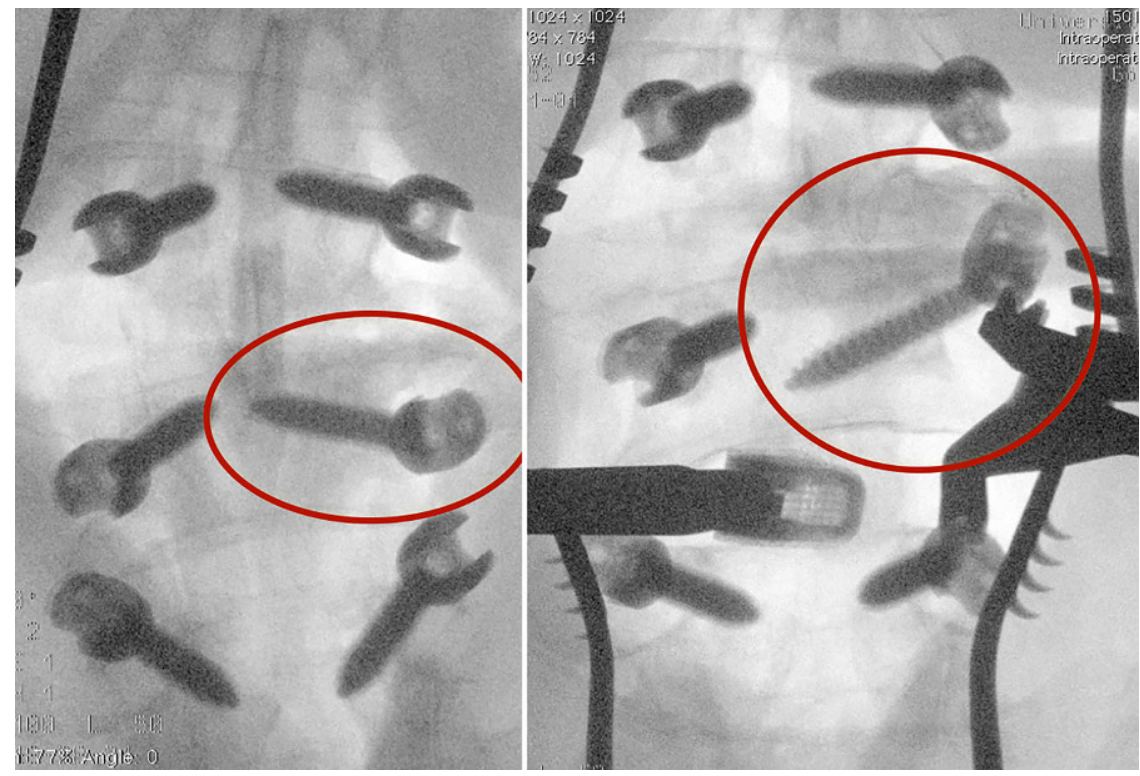

Abb. 1 ॥ Intraoperativ kann sich eine Schraubenlockerung sowohl makroskopisch als auch im Rahmen der durchgeführten Bildwandlerkontrollen zeigen. Des Weiteren kann das intraoperative Eindrehmoment der Pedikelschraube einen Hinweis auf eine insuffiziente Verankerung geben. Im Fall einer gelockerten/ausgerissenen Schraube sollte diese zunächst vorsichtig entfernt werden
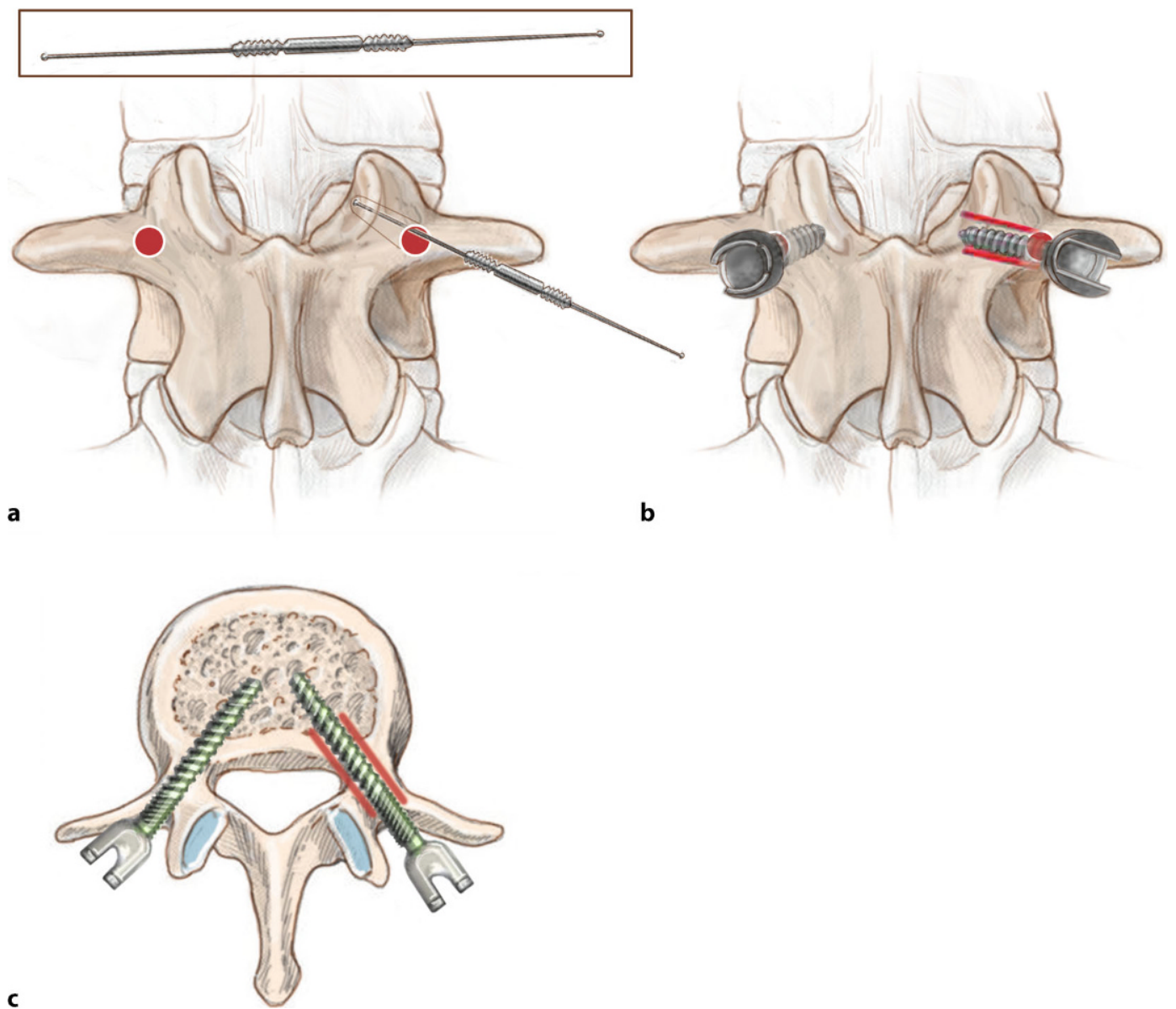

Abb. $2<$ Anschließend sollte der Schraubenkanal mit der Pedikelsonde vollständig ausgetastet werden und insbesondere auf eine Perforation in den Spinalkanal untersucht werden 


\section{Operative Techniken}

\section{Zementaugmentation}

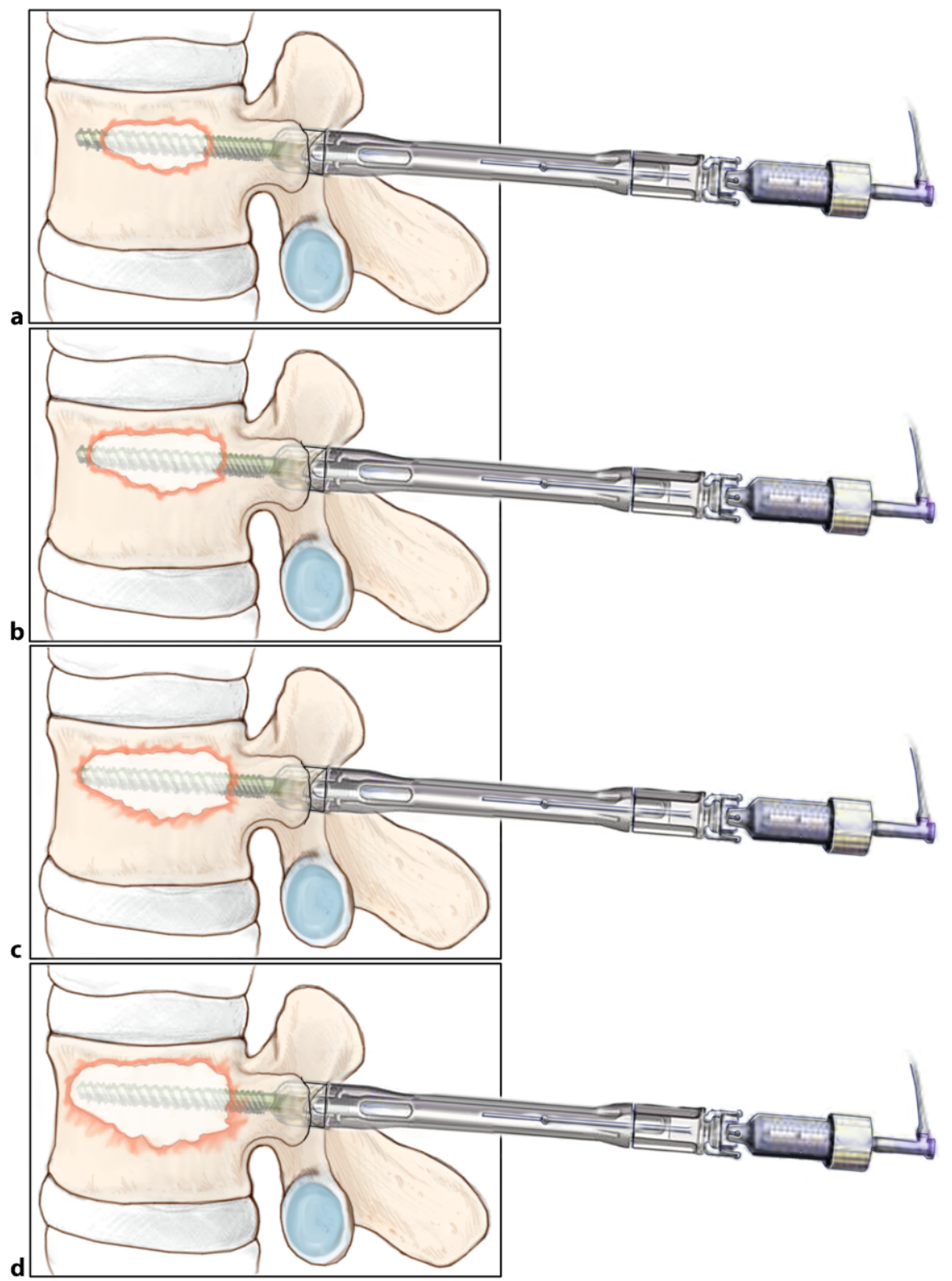

Abb. 3 A Bei älteren Patienten oder bei Vorliegen einer Osteopenie/Osteoporose sollte eine nachträgliche Zementaugmentation der Pedikelschraube durchgeführt werden. Die Augmentation kann auf zwei unterschiedliche Art und Weisen erfolgen. Bei Vorhandensein eines kanülierten, perforierten Schraubensystems kann nun eine augmentierbare Pedikelschraube, wenn möglich mit einem um $1 \mathrm{~mm}$ größeren Schraubendurchmesser, eingebracht werden. Die Schraubenlage sollte mit dem Bildwandler im a.-p.- und seitlichen Strahlengang kontrolliert werden. Anschließend erfolgt die Augmentation mit Knochenzement 


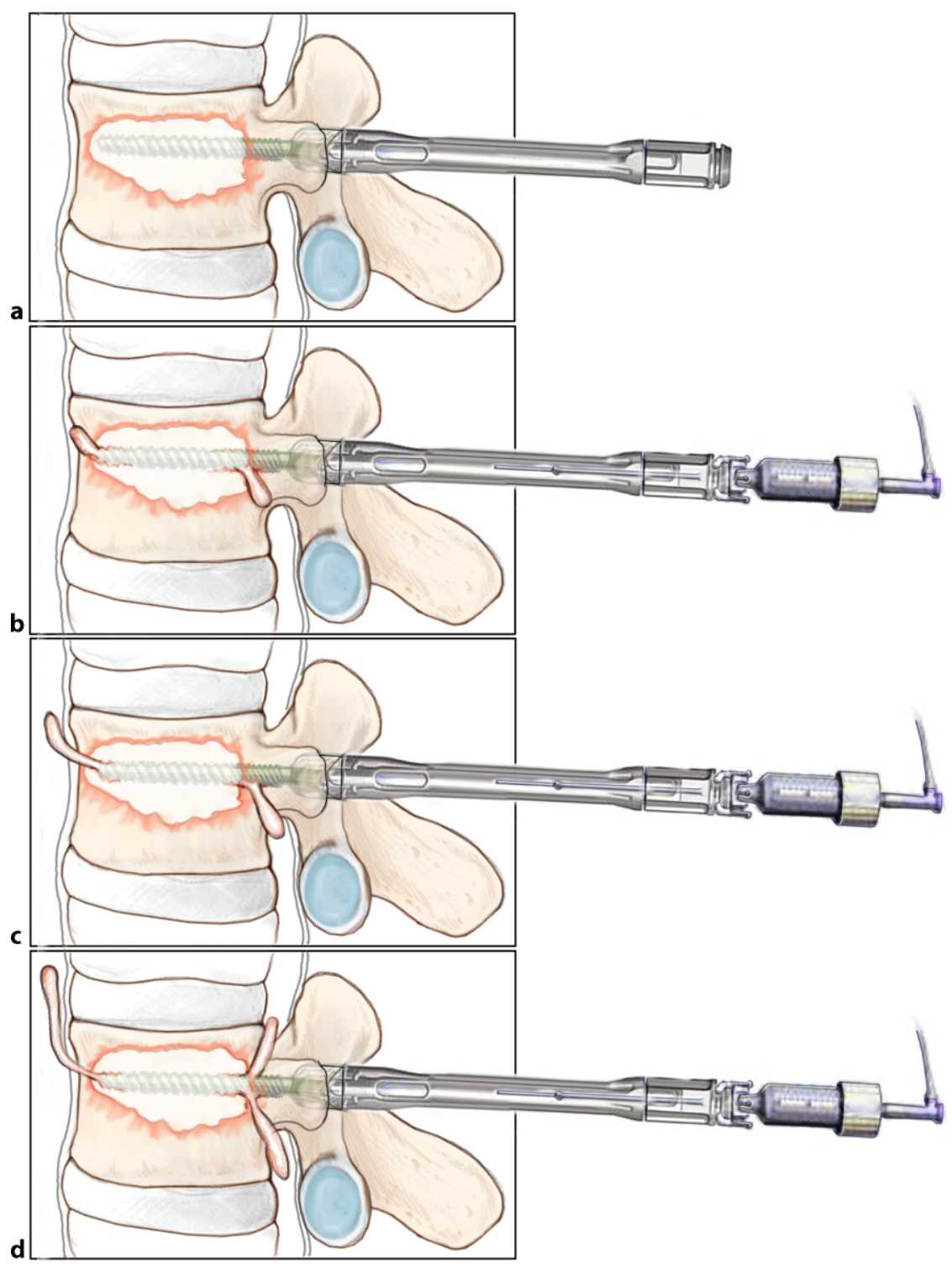

Abb. 4 A Bei der Zementapplikation ist zu beachten, dass das injizierte Volumen das Volumen einer Augmentation vor Lockerung übersteigen kann, da bereits ein größeres Cavum durch die Lockerung der eingebrachten Schraube entstanden ist. Das Volumen beträgt in etwa 2-5 $\mathrm{ml}$. Während der Augmentation sollten regelmäßige radiologische Kontrollen durchgeführt werden, um eineZementleckage oder eine Embolie zu verhindern 


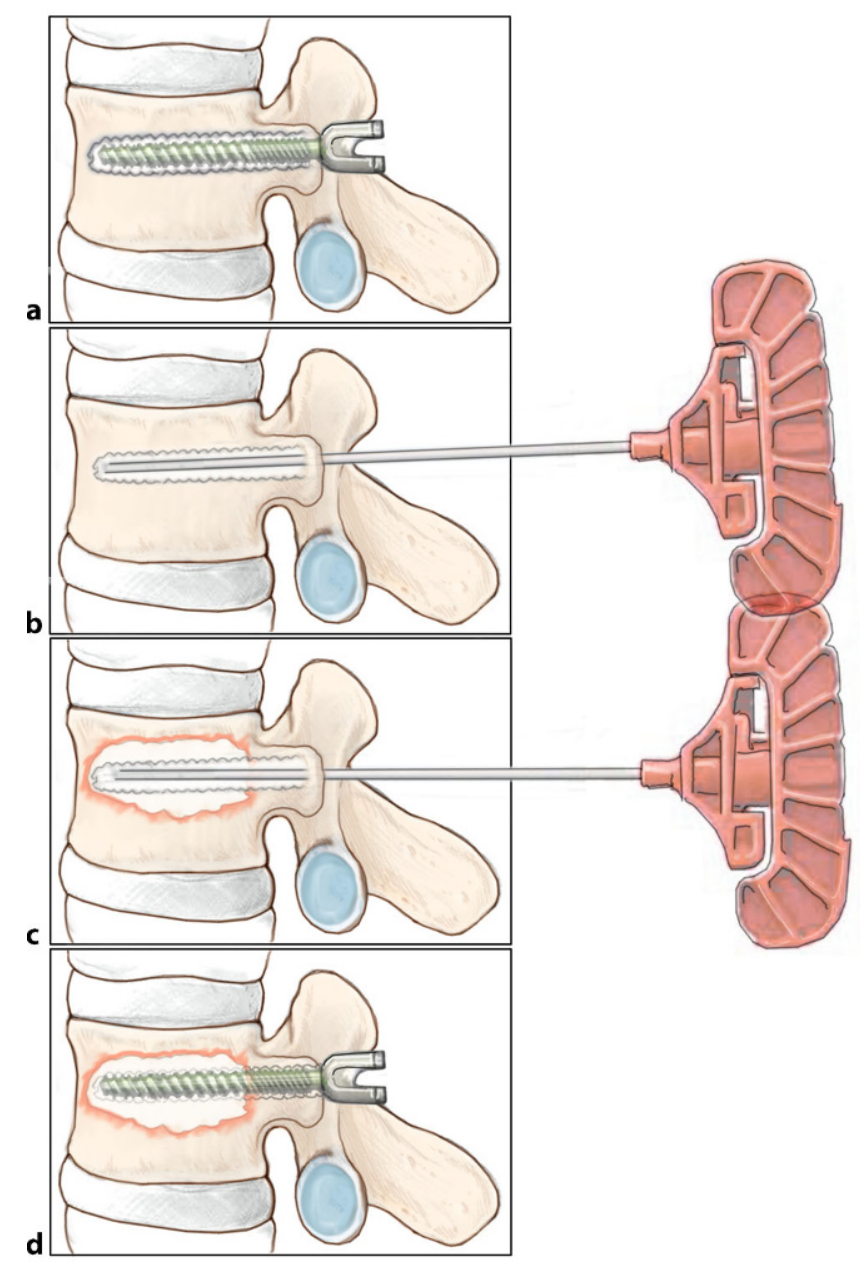

Abb. $5<$ Sollte

kein kanüliertes System zu Verfügung stehen, kann der entstandene Defekt im Wirbelkörper im Sinne einer Vertebroplastie aufgefüllt werden. Hierfür ist es essenziell, den Schraubenkanal auf eine mögliche Perforation zu überprüfen. Liegt eine Perforation in den Spinalkanal vor, sollte dieses Verfahren nicht angewendet werden. Nach Entfernung der einliegenden Schraube und Austasten des Schraubenkanals kann über eine Jamshidi-Nadel der entstandene Defekt im Wirbelkörper mit Polymethylmethacrylat-(PMMA-)Zement aufgefüllt werden. Anschließend wird die Pedikelschraube in den noch weichen Zement eingedreht 


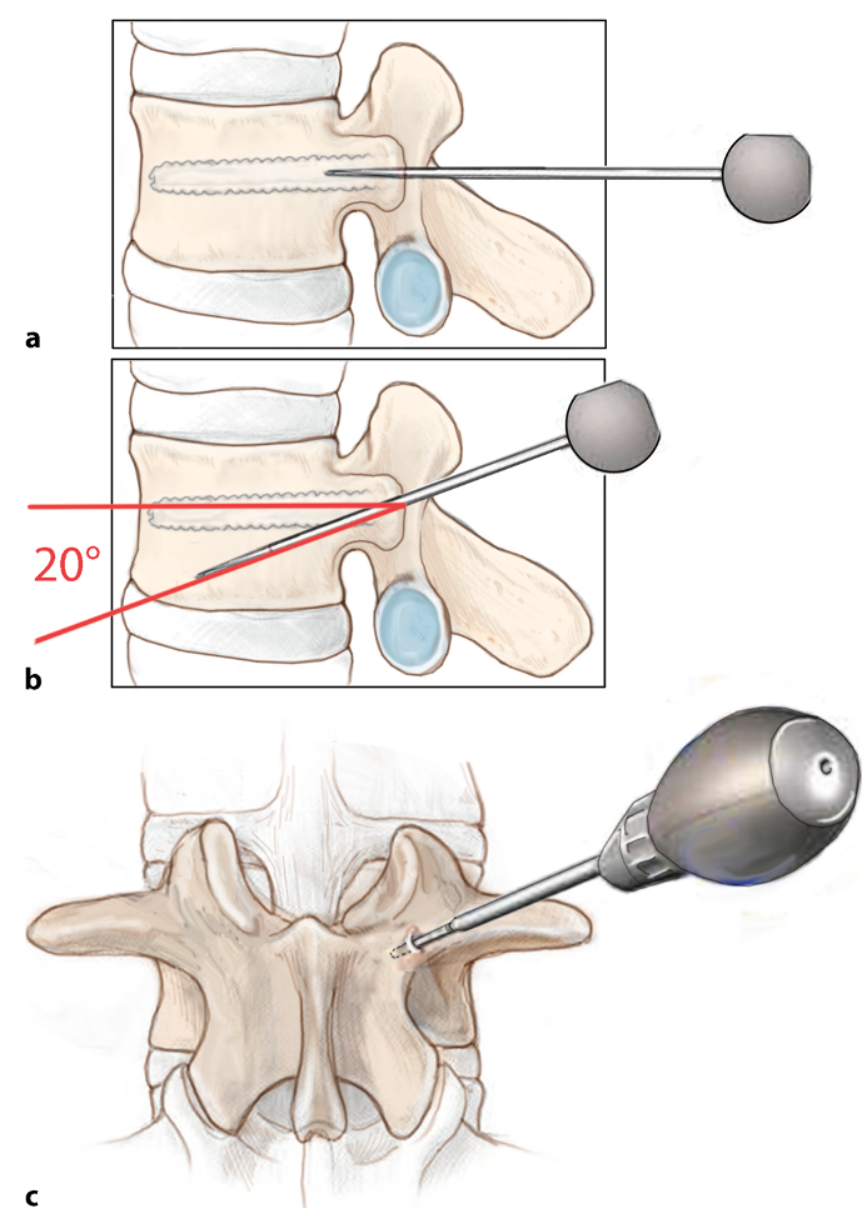

Abb. 6 \& Insbesondere bei jüngeren Patienten mit adäquater Knochendichte kann versucht werden, die gelockerte Pedikelschraube in einer alternativen Trajektorie, etwa in Cauda-Technik, einzubringen. Wie oben beschrieben, sollte auch hier zunächst die gelockerte Schraube entfernt werden und der Kanal sorgfältig ausgetastet werden. Anschließend Eingehen mit dem spitzen Pfriem bis zur Wirbelkörperhinterkante. Es sollte radiologisch kontrolliert werden, dass der Pfriem den gesamten Pedikel passiert hat, um eine Perforation zu vermeiden. Anschließend kann der Pfriem nach kaudal und medial geschwenkt werden und ein alternativer Schraubenkanal im Bereich des intakten Knochens geschaffen werden. Die Cauda-Schraube verläuft in Abhängigkeit des Wirbelkörpers 0-10 nach medial gerichtet und etwa $20^{\circ}$ nach kaudal gerichtet. Eine weitere Möglichkeit der Revision einer intraoperativ gelockerten Pedikelschraube ist die Verwendung eines größeren Schraubendurchmessers. Dies ist aus unserer Sicht insbesondere bei gelockerten Schrauben ohne wesentliche Destruktion im Wirbelkörper, ohne relevante Dislokation und bei Patienten mit allenfalls geringfügig reduzierter Knochendichte indiziert

\section{Postoperative Behandlung}

- Direkt postoperative Kontrolle der peripheren Durchblutung, Motorik und Sensibilität

- Mobilisation ab dem 1. postoperativen Tag

- Radiologische Kontrolle mit konventionellem Röntgenbild und ggf. CT

- Funktionelle Nachbehandlung unter Vermeidung des Hebens schwerer Lasten $>5 \mathrm{~kg}$

- Medikamentöse Behandlung der Osteoporose

- Ggf. Materialentfernung im Verlauf (>6 Monate)

\section{Fehler, Gefahren, Komplikationen}

- Zementleckage und -embolie

- Verletzungen benachbarter Strukturen, insbesondere Spinalnerv, Dura, Myelon

- Materialfehllage, Materiallockerung

\section{Ergebnisse}

In einer von den Autoren durchgeführten biomechanischen Studie wurde die nachträgliche Augmentation nach intraoperativer Schraubenlockerung untersucht. Es wurden 14 humane LWK 2 Wirbelkörper (Durchschnittsalter $72 \pm 14$ Jahre) in die Studie eingeschlossen. Bei allen Präparaten lag eine Osteopenie/Osteoporose vor (Mittelwert BMD [,bone mineral density“] $72 \pm 22 \mathrm{mg} / \mathrm{cm}^{3}$; qCT). Es erfolgte ein sinusförmiger Dauerbelastungstest $(50-100 \mathrm{~N})$ mit einer zyklischen Laststeigerung um $0,1 \mathrm{~N}$ bis zur Lockerung der Schrauben. Die Testung erfolgte zunächst auf der einen Seite mit augmentierten und auf der anderen Seite mit nichtaugmentierten Pedikelschrauben. Nach eingetretener Lockerung der eingebrachten Schrauben, erfolgte eine nachträgliche Augmentation der gelockerten, nichtaugmentierten Schraube und es wurde ein erneuter Dauerbelastungstest durchgeführt. Die injizierten Zementvolumina richteten sich nach dem radiologischen Befund während der Augmentation und betrugen im Fall der initialen Augmentation 2-3 ml 
und im Falle der Augmentation nach Lockerung 2-5 ml. Ausgewertet wurden die gemessenen Steifigkeiten sowie die Zyklen und die Kraft bis zum Versagen.

Durch die initiale Augmentation zeigte sich ein Anstieg der Versagenslast um $45 \%$ verglichen mit den nichtaugmentierten Schrauben (nichtaugmentiert $207 \mathrm{~N}, 1078$ Zyklen vs. augmentiert 301 N, 2012 Zyklen; $p=0,009$ ). Die nach bereits eingetretener Lockerung augmentierten Schrauben zeigten einen Anstieg der Versagenslast um 79\% verglichen mit den nichtaugmentierten Schrauben $(370 \mathrm{~N}, 2728$ Zyklen; $p=0,001)$. Der weitere Anstieg der Versagenslast bei den nach Lockerung augmentierten Schrauben ist vermutlich darauf zurückzuführen, dass durch die Lockerung ein Cavum für den Zement geschaffen wird und die instabile Spongiosa bereits bricht. Anschließend kann ein größeres Zementvolumen appliziert werden und durch die somit größere Auflagefläche kommt es zum Anstieg der Versagenslast. Der Unterschied der Last bis zum Versagen zwischen den augmentierten und den nach Lockerung augmentierten Schrauben ist jedoch nicht signifikant $(p=0,056)$. Es ist daher zu schlussfolgern, dass die nachträgliche Augmentation einer intraoperativ gelockerten Schraube möglich und bezüglich der Schraubenstabilität mindestens gleichwertig verglichen mit der Augmentation nichtgelockerter Pedikelschrauben ist.

In unserem eigenen Patientenkollektiv aus dem Zeitraum April 2016 bis August 2018 zeigte sich bei 11 Patienten eine intraoperative Schraubenlockerung. Definiert wurde die Lockerung hierbei als radiologische Dislokation einer eingebrachten Schraube beziehungsweise klinisch signifikante Lockerung in Form einer wackelnden Schraube im Wirbelkörper ohne relevantes Eindrehmoment. Diese Lockerungen behandelten wir in 6 Fällen mit einer nachträglichen Augmentation über fenestrierte Pedikelschrauben und in 5 Fällen durch Auswahl eines dickeren Schraubendurchmessers. In der postoperativen Kontrolle nach 6 Wochen zeigte sich keine dieser Schrauben radiologisch erneut gelockert (konventionelles Rönt-
gen-CT) und die Patienten berichteten nicht über eine erneut zunehmende Beschwerdesymptomatik.

Die Entscheidung, ob eine nachträgliche Augmentation der gelockerten Schrauben erfolgen oder diese durch eine dickere Schraube ersetzt werden sollte, richtet sich - nach unserer Meinung nach der vorliegenden Knochendichte. Kiner et al. beschrieben in ihrer biomechanischen Untersuchung, dass die Wahl einer dickeren Pedikelschraube der Zementaugmentation überlegen sei [9]. Jedoch erfolgte die Zementaugmentation nicht über fenestrierte Schrauben, welche eine signifikant höhere Stabilität verglichen mit per Vertebroplastie augmentierten Schrauben aufweisen [5]. Des Weiteren wurde in den Experimenten die Knochendichte, welche aus unserer Sicht den entscheidenden Faktor darstellt, nicht ausreichend berücksichtigt und die Testung erfolgte nicht bis zur Schraubenlockerung.

Zusammenfassend sollte aus unserer Sicht im Fall einer intraoperativen Schraubenlockerung immer eine an die Pedikelweite optimierte Schraubendicke gewählt werden und in Abhängigkeit der vorliegenden Knochendichte über eine zusätzliche Augmentation entschieden werden.

\section{Korrespondenzadresse}

\section{Dr. Lukas Weiser}

Klinik für Unfallchirurgie, Orthopädie und Plastische Chirurgie, Universitätsmedizin Göttingen

Robert-Koch-Straße 40, 37099 Göttingen, Deutschland

Lukas.Weiser@med.uni-goettingen.de

\section{Einhaltung ethischer Richtlinien}

Interessenkonflikt. L. Weiser, S. Sehmisch, L. Viezens und W. Lehmann geben an, dass kein Interessenkonflikt besteht.

Für diesen Beitrag wurden von den Autoren keine Studien an Menschen oder Tieren durchgeführt. Für die aufgeführten Studien gelten die jeweils dort angegebenen ethischen Richtlinien.

Open Access. Dieser Artikel wird unter der Creative Commons Namensnennung 4.0 International Lizenz (http://creativecommons.org/licenses/by/4.0/deed. de) veröffentlicht, welche die Nutzung, Vervielfältigung, Bearbeitung, Verbreitung und Wiedergabe in jeglichem Medium und Format erlaubt, sofern Sie den/die ursprünglichen Autor(en) und die Quelle ordnungsgemäßnennen, einen Linkzur Creative Commons Lizenz beifügen und angeben, ob Änderungen vorgenommen wurden.

\section{Literatur}

1. Galbusera F, Volkheimer D, Reitmaier S, BergerRoscher N, Kienle A, Wilke HJ (2015) Pedicle screw loosening: A clinically relevant complication? Eur Spine J24(5):1005-1016

2. Halvorson TL, Kelley LA, Thomas KA, WhitecloudTS 3rd, CookSD (1994) Effects of bone mineral density on pedicle screw fixation. Spine (Phila Pa 1976) 19(21):2415-2420

3. Soshi S, Shiba R, Kondo H, Murota K (1991) An experimental study on transpedicular screw fixation in relation to osteoporosis of the lumbar spine. Spine (Phila Pa 1976) 16(11):1335-1341

4. Weiser L, Huber G, Sellenschloh K, Viezens L, Püschel K, Morlock MM, Lehmann W (2017) Insufficient stability of pedicle screws in osteoporotic vertebrae: Biomechanical correlation of bone mineral density and pedicle screw fixation strength. Eur Spine J. https://doi.org/10.1007/s00586-0175091-x

5. Kueny RA, Kolb JP, Lehmann W, Püschel K, Morlock MM, Huber G (2014) Influence of the screw augmentation technique and a diameter increase on pedicle screw fixation in the osteoporotic spine: Pullout versus fatigue testing. Eur Spine J 23(10):2196-2202

6. Leichtle Cl, Lorenz A, Rothstock S, Happel J, Walter F, Shiozawa T, Leichtle UG (2016) Pull-out strength of cemented solid versus fenestrated pedicle screws in osteoporotic vertebrae. Bone Joint Res 5(9):419-426

7. Weiser L, Huber G, Sellenschloh K, Viezens L, Puschel K, Morlock MM, Lehmann W (2018) Time to augment?! Impact of cement augmentation on pedicle screw fixation strength depending on bone mineral density. Eur Spine J 27(8):1964-1971

8. Kocak T, Daxle M, Reichel H, Lattig F (2013) Alternative technique of cement augmentation of loosened pedicle screws-Technical note and presentation of two cases. Acta Chir Orthop Traumatol Cech 80(1):89-91

9. Kiner DW, Wybo CD, Sterba W, Yeni YN, Bartol SW, Vaidya R (2008) Biomechanical analysis of different techniques in revision spinal instrumentation: larger diameter screws versus cement augmentation. Spine (Phila Pa 1976) 33(24):2618-2622

10. Matsukawa K, Taguchi E, Yato $Y$, Imabayashi H, Hosogane N, Asazuma T, Nemoto K (2015) Evaluation of the fixation strength of pedicle screws using cortical bone trajectory: What is the ideal trajectory for optimal fixation? Spine (Phila Pa 1976) 40(15):E873-E878

11. Matsukawa K, Yato $Y$, Imabayashi $H$, Hosogane N, Asazuma T, Nemoto K (2015) Biomechanical evaluation of the fixation strength of lumbar pedicle screws using cortical bone trajectory: A finite element study. J Neurosurg Spine 23(4):471-478

12. Yu T, Zhang X, Liu J, Li S, Shan Z, Fan S, Zhao F (2018) Superior cortical screw in osteoporotic lumbar vertebrae: $A$ biomechanics and microstructurebased study. Clin Biomech (Bristol, Avon) 53:14-21

13. Janssen I, Ryang YM, Gempt J, Bette S, Gerhardt J, Kirschke JS, Meyer B (2017) Risk of cement leakage and pulmonary embolism by bone cement-augmented pedicle screw fixation of the thoracolumbar spine. Spine J 17(6):837-844 